\title{
Correction to: Auditing data resolves systemic errors in databases and confirms mycorrhizal trait consistency for most genera and families of flowering plants
}

\author{
Mark C. Brundrett ${ }^{1}$
}

Published online: 23 December 2021

๑) Springer-Verlag GmbH Germany, part of Springer Nature 2021

\section{Correction to: Mycorrhiza (2021) 31:671-683 \\ https://doi.org/10.1007/s00572-021-01051-4}

The Publisher regret that the data of Table 1 in the published version of the above article were incorrect. The correct data of Table 1 should have been presented as follows:

The original article can be found online at https://doi.org/10.1007/ s00572-021-01051-4.

Mark C. Brundrett

brundrett@uwa.edu.au

1 School of Biological Sciences, University of Western Australia, 35 Stirling Highway, Crawley, WA 6009, Australia 
Table 1 Factors that determine mycorrhizal data quality listed in increasing levels of complexity for each category. See Box 1 for limitations to use of data

A. Sample sources, quality and quantity:

1. Random samples of roots excavated from natural habitats at a single time and location.

2. Excavated root samples taken from entire small plants or by carefully tracing roots back to the base of plants.

3. As in 2 above, but including substantial replication, both in space and time to ensure active mycorrhizal roots are examined.

4. Plants cultivated in natural soils harvested when roots are growing actively (bioassays).

5. Plant cultivated as above, but with specific mycorrhizal fungi inoculated using spores or living cultures.

6. Time course studies showing the sequence of mycorrhizal interface formation and senescence.

B. Methodology for observations and measurements:

1. Observations of suspected mycorrhizal fungi fruiting near plants (e.g. AM spores in the rhizosphere or putative EM fungi fruiting near trees).

2. Superficial examination of roots in situ to record arrangement, colour and smell.

3. Stereomicroscopic examination of whole unprocessed roots to examine their shape, branching patterns and colour (e.g. EM short roots).

4. Microscopic examination of whole cleared roots (or other structures) to reveal details of internal hyphal arrangement using samples with adequate quality and quantity.

5. Detailed anatomical studies using sectioned roots and selective stains by light and/or electron microscopy.

6. Examining samples of different ages to establish the time course and duration of mycorrhizal fungus activity.

7. Physiological studies linking mycorrhizal formation to benefits using carefully regulated soil nutrient levels and appropriate controls.

8. Measuring mycorrhizal development and functioning across a wide range of soil fertility levels (nutrient response curves).

9. Detailed physiological studies measuring plant growth responses, bi-directional nutrient transfer, metabolic activity, nutrient accumulation, etc.

10. Metabolomic and genomic studies of active mycorrhizas.

The original article has been corrected.

Publisher's Note Springer Nature remains neutral with regard to jurisdictional claims in published maps and institutional affiliations. 